\title{
Unusual variation in musculocutaneous nerves
}

\author{
Bhattarai $\mathbf{C}^{1}$, Poudel PP ${ }^{1}$ \\ ${ }^{1}$ Lecturer, Department of Anatomy, Manipal College of Medical Sciences, Pokhara, Nepal
}

\begin{abstract}
Background: Variations in origin, course, branching pattern, termination and connections of the musculocutaneous nerve in the arm are not as uncommon as was once thought. Variational study of nerve in Nepalese is very less and can be of surigical-clinical importance. This opinion led us to performing this study.

Objectives: The aim of this study was to a study in Nepalese which could be important for clinical investigation and the surgical treatment of peripheral nerve injury.

Materials and methods: This study was carried out in Manipal College of medical sciences (MCOMS). Dissection of formalised 16 cadavers ( $\mathrm{n}=32$ upper limbs) were carried out using normal dissection kit with the help of standard dissection manual in two and half years.

Results: The study revealed the variation in course, branching pattern and termination of musculocutaneous nerve in $6.25 \%$ of cases unilaterally on the right side. No statistically significant differences by gender and side were observed.

Conclusion: This present study provides the evidence of variation of musculocutaneous nerve in Nepalese. The knowledge of the anatomical variations of the peripheral nerve system can help explain an incomprehensible clinical sign.
\end{abstract}

Key words: Musculocutaneous nerve, Median nerve, Brachial plexus, Peripheral nerve repair.

$\mathrm{M}$

usculocutaneous nerve arises from the lateral cord (C5-7), opposite the lower border of Pectoralis minor at the distal third of axillary fossa as a terminal branch, usually pierces the Coracobrachialis and descends inferolaterally between the Biceps brachii and Brachialis and terminates as Lateral cutaneous nerve of forearm ${ }^{1}$. Several variations on musculocutaneous nerves have been well described by many authors. However, our study is concerned about the variation in Nepalese and its magnitude. We have also emphasised on clinical importance. We studied the course of musculocutaneous nerve during routine dissection of formalin embalmed 16 cadavers (32 upper limbs) in a period of two and half years. We believe that study of variation in the peripheral nerves noticed in cadaveric dissections should be included in surgical training programs, even if they are not necessary for inclusion in routine anatomy education in medical schools.

\section{Materials and methods}

This study was carried out on formalin embalmed 16 cadavers ( $n=32$ upper limbs) in a period of two and half years. Procedures for the study were performed by dissection using regular dissection kit and following the standard dissection manual. Study was analysed by comparing with normal standard gross origin, courses, and branches as stated in the Gray's Anatomy. Numbers of abnormal observation were calculated in percentage with reference to total numbers of observations and were put into statistical analysis.

Results

Variations in courses, branching pattern and connections of Musculocutaneous nerves in the arm were observed. These variations were seen in $6.25 \%$ of cases unilaterally on the right side out of total 32 upper limbs. No statistically significant differences by gender and side were observed.

In $3.125 \%$ of cases on the right side (fig1), it was found that the nerve after piercing Coracobrachialis ran downwards between short head of Biceps brachii and medial intermuscular septa lateral to brachial artery. The nerve then terminated by joining with Median nerve at the level of junction of distal and middle third of medial side of arm. We also observed the Lateral cutaneous

\footnotetext{
Correspondence
}

Ms. Chacchu Bhattarai

Lecturer, Department of Anatomy

Manipal College of Medical Sciences,

Mahendragufa Marga, Deep Height-16,

Pokhara, Nepal

E-mail: chachhu_anat@yahoo.com, chachhubhattarai@gmail.com 
nerve of forearm as a thin branch splitting from the main trunk just proximal to its termination and passing deep to unusual third head of Biceps brachii downward and laterally.

In remaining $3.125 \%$ of cases (fig2), the nerve gave two thick branches one lateral and another medial at the level of surgical neck of Humerus just before piercing the Coracobrachialis. The lateral branch was found piercing the Coracobrachialis and terminating

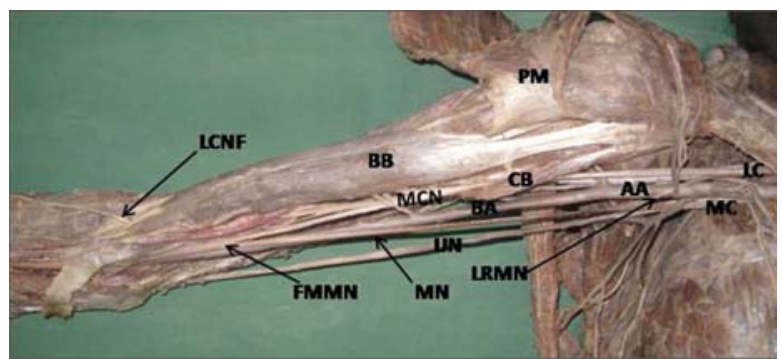

Fig 1: Variation in pattern of the right Musculocutaneous Nerve

LC = Lateral Cord of Brachial Plexus

$\mathrm{MC}=$ Medial Cord of Brachial Plexus

LRMN $=$ Lateral Root of Median Nerve

$\mathrm{AA}=$ Axillary Artery

$\mathrm{BA}=$ Brachial Artery

$\mathrm{PM} \quad=$ Pectoralis Major

$\mathrm{BB}=$ Biceps Brachii

$\mathrm{CB}=$ Coracobrachialis

$\mathrm{MCN}=$ Musculocutaneous Nerve

$\mathrm{MN}=$ Median Nerve

FMMN $=$ Fusion of Musculocutaneous nerve with Median Nerve

LCNF $=$ Lateral Cutaneous Nerve of Forearm

\section{Discussion}

The study of variation of Musculocutaneous nerve in our country is very less. Our study found unusual courses, branching pattern and termination of Musculocutaneous nerve unilaterally. This study provides essential information to surgeons about the possible percentage of variation regarding this nerve in Nepalese population. Variation in brachial plexuses along with its terminal branches have been revealed by many authors. Studies by Nakatani et al. revealed three variations in which the musculocutaneous nerve did not pierce the coracobrachialis ${ }^{2}$. Le minor described a case of a lateral small branch that first pierced the coracobrachialis muscle and then one of its terminal branches, joining the medial root forming the medial nerve $^{3}$. Iwamoto et al reported a communicating branch from the musculocutaneous to median nerve ${ }^{4}$. Tsikaras into muscular branch to Biceps brachii and Brachialis. The medial branch connected with median nerve at the junction of upper and middle third of the arm. Regarding muscular branch to Coracobrachialis it was seen as a small twig at the level of origin of main trunk. Lateral cutaneous nerve of forearm in this observation, revealed originating from the median nerve at the level of middle and lower third of arm and coursing downward and laterally between normal fused belly and unusual third head of Biceps brachii.

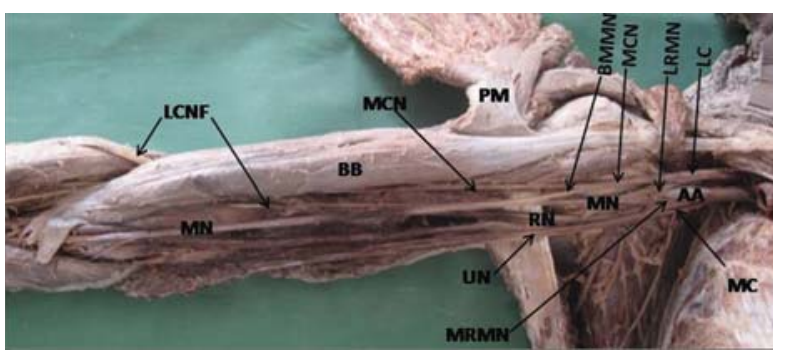

Fig 2: Variation in pattern of the right Musculocutaneous Nerve

$\mathrm{LC}=$ Lateral Cord of Brachial Plexus

$\mathrm{AA}=$ Axillary Artery

$\mathrm{MC}=$ Medial Cord of Brachial Plexus

LRMN = Lateral Root of Median Nerve

MRMN $=$ Median Root of Median Nerve

$\mathrm{MCN}=$ Musculocutaneous Nerve

$\mathrm{MN}=$ Median Nerve

$\mathrm{BMMN}=$ Branch of Musculocutaneous Nerve to Median Nerve

$\mathrm{RN}=$ Radial Nerve

$\mathrm{UN}=$ Ulnar Nerve

$\mathrm{PM}=$ Pectoralis Major Muscle

$\mathrm{BB}=$ Biceps Brachii

LCNF $=$ Lateral Cutaneous Nerve of Forearm

et al. revealed that the musculocutaneous nerve arose from the median nerve unilaterally in a male cadaver ${ }^{5}$. Even the absence of musculocutaneous nerve, variation in the brachial plexus and also the unusual course, distribution, patterns of connections with other nerves has also been observed by authors $6,7,8,9,10,11$.

From clinical point of view, such variations are of great importance during flap dissections, posttraumatic evaluations of the arm or peripheral nerve repair. After trauma to the arm, when the median nerve seems to be surgically intact, the fibers coursing in the musculocutaneous nerve may be damaged. While flap dissections an unexpected damage could also occur to the musculocutaneous nerve ${ }^{6}$. Different communications involving musculocutaneous nerve 
are important in repairs for trauma to the shoulder and also in understanding of the musculocutaneous nerve dysfunction ${ }^{13}$. A more precise knowledge than that found in classical anatomical texts is necessary for clinical investigation and the surgical treatment of peripheral nerve injury ${ }^{14}$. Comprehensive look for the peripheral nerve distribution, course, and origin in arm should be well known by the medical person. Nerve injuries about the shoulder and proximal upper limb are uncommon but important causes of pain and dysfunction in athletes ${ }^{15}$. Peripheral nerve stimulation in the practice of brachial plexus anaesthesia has also been revealed ${ }^{16}$.

Thus this study of unusual courses, branching pattern and termination of musculocutaneous nerve is of clinical importance during flap dissections, post traumatic evaluation of the arm or peripheral nerve repair and even for peripheral nerve stimulation in practice of anaesthesia.

\section{Conclusion}

The present study describes unilateral variations of Musculocutaneous nerve in Nepalese. Because each peripheral nerve is a collection of nerve fibres bound together by connective tissue, unusual variation could be possible. In summary, anatomists and surgical practitioners should be aware of these variations so as to avoid unnecessary damages during dissection as well as surgery. Knowledge about this variation in origin, course, and pattern of branching, distribution will be useful for surgeons to avoid injury while performing surgeries in arm. We believe that this possibility of variation of the peripheral nerve noticed in cadaveric dissections should be included in surgical training programs, even if they are not necessarily for inclusion in routine anatomy education in medical schools.

\section{References}

1. Johnson D, Harold E. Pectoral girdle, Shoulder Region and Axilla. In: Standring S, editor. Gray's Anatomy. 39th ed. New York: Elsevier Churchill Livingstone; 2001. p. 848.

2. Nahtani T, Miukami S, Tanah S. Three cases of the musculocutaneous nerve not perforating the coracobrachialis muscle. Kaibogakukshi.1997;72:191-4.

3. Le Minor IM. A rare variation of the median and musculocutaneous nerves in man. Arch Annt Histol Embryul. 1990;73:33-42.

4. lwmoto S, Kimura K, Takahashi Y, Konishi M. Some aspects of the communicating branch between the musculocutaneous and median nerves in man. Okajimas Folia Anat Jpn. 1990;67:47-52.

5. Tsikaas PD, Agiabasis AS, Hytiroglou PM. A variation in the formation of the brachial plexus characterised by the absence of C8 and TI fiber in the trunk of the median nerve. Bullhsoc Anat (Nancy). 1983;67:501-5.

6. Ibrahim $\mathrm{CH}$, Adnan E, Cem DC. Variation between Median and Musculocutaneous Nerves. The Internet Journal of Surgery. 2005;6(1).

7. Song WC, Jung HS, Kim HJ, Shin C, Lee BY, Koh KS. A variation of the musculocutaneous nerve absent. Yonsei Med J. 2003;44:1110-3.

8. Gümüsburun E, Adigüzel E. A variation of the brachial plexus characterized by the absence of the musculocutaneous nerve: a case report. Surg Radiol Anat. 2000;22:63-5.

9. Saeed M, Rufai AA. Median and musculocutaneous nerves: variant formation and distribution. Clin Anat. 2003;16:453-7.

10. Gümüşalan Y, Yazar F, Ozan H. Variant innervation of the coracobrachialis muscle and unusual course of the musculocutaneous nerve in man. Kaibogaku Zasshi.1998;73:269-72.

11. Choi D, Rodríguez-Niedenführ M, Vázquez T, Parkin I, Sañudo JR. Patterns of connections between the musculocutaneous and median nerves in the axilla and arm. Clin Anat. 2002;15:11-7.

12. Meshram S, Rawlani S, Chimurkar VK, Shah $\mathrm{MM}$ and Belsare M. Bilateral variations in nerves and vascular pattern in the upper limb. 54th National Conference of The Anatomical Society of India; 2006; India: Anatomical Society of India; 2007.p.1 - 55.

13. Chitra R. Various types of intercommunications between musculocutaneous and median nerves: An analytical study. Annals of Indian Academy of Neurology. 2007;10:100-4.

14. Linell EA. The distribution of nerves in the upper limb, with reference to variabilities and their clinical significance. Journal of Anatomy. 1921;55:79-112.

15. McInnis KC, Krivickas LS. Peripheral Nerve Injuries of the Shoulder and Upper Arm. Nerve and Vascular injuries in sports medicine. New York: Springer; 2009. p. 87-111.

16. Andres JD, Blanch XS. Peripheral nerve stimulation in the practice of brachial plexus anesthesia: A review. Regional Anesthesia and Pain Medicine. 2001;26:478-83. 